\title{
The Effect of Personality on Psychological Responses Induced by Emotional Stimuli for Children
}

\author{
Eun Hye Jang', Youngji Eum², Suk-Hee Kim³, Jin-Hun Sohn² \\ ${ }^{1}$ ETRI, Bio-health IT Convergence Research Department, Daejeon, 305-700 \\ ${ }^{2}$ Chungnam National University, Department of Psychology, Daejeon, 305-764 \\ ${ }^{3}$ Wonkwang University, Department of Professional Counseling \& Psychotherapy, Iksan, 570-749
}

\author{
Corresponding Author \\ Jin Hun Sohn \\ Chungnam National University, \\ Department of Psychology, Daejeon, \\ 305-765 \\ Mobile : +82-10-9350-6369 \\ Email : jhsohn@cnu.ac.kr
}

Received : April 29, 2014

Revised : May 14, 2014

Accepted: August 29, 2014

Copyright@2014 by Ergonomics Society of Korea. All right reserved.

(c) This is an open-access article distributed under the terms of the Creative Commons Attribution Non-Commercial License (http:// creativecommons.org/licenses/by-nc/3.0/), which permits unrestricted non-commercial use, distribution, and reproduction in any medium, provided the original work is properly cited.
Objective: The aim of this study is to identify the relationship between personality and psychological responses induced by emotional stimuli (happiness, sadness, anger, boring and stress) for children.

Background: Many researches are interested in assertion that there is close correlation between personality and emotion. The relationship between personality and emotion needs to be studied in view of the extended integration, not in view of respective property, because personality is deeply ingrained, and the relatively enduring patterns of thought, feeling and behavior and emotion can take advantage of individual differences in sensitivities to situational cues and predispositions to emotional state. In particular, studies on the personality and emotion for children are necessary in that childhood is an important period for formation of their personality and emotion expression and regulation.

Method: Prior to the experiment, we made parents of 94 children rate personalities of their children, based on Korean Personality Inventory for Children (K-PIC). Results of 64 children without missing answers to all questions were analyzed. 64 children were exposed to five emotional stimuli and were asked to report the classification and intensity of their experienced emotion.

Results: Children were classified into two groups of the lower $25 \%$ and higher $25 \%$ scores in twenty sub-scales of K-PIC, and psychological responses to five emotional stimuli between two groups were compared. Accuracy of emotion experienced by emotional stimuli showed a significant difference between the two groups, the lower and higher scores in Hyperactivity and Adjustment. Also, there was a significant difference in the intensity of experienced emotions between the two groups in Intellectual Screening and Psychosis.

Conclusion: Our result has shown that hyperactivity, adjustment, intellectual screening and psychosis influence the accuracy and intensity of emotional responses.

Application: This study can offer a guideline to overcome methodological limitation of emotion studies for children and help researcher basically understand and recognize human emotion in $\mathrm{HCl}$.

Keywords: Children, Personality, Emotion

\section{Introduction}

Personality is a complex of thought, emotion and behavior demonstrated, as individual's 
inner psychological and physical systems are dynamically organized. Personality represents individual's unique characteristics and is a consistent pattern. Personality is individual's inner process including psychological mechanism's emotional, motivational and cognitive processes affecting individual's characteristic behavior, feeling and thought (Bueger and Caldwell, 2000). Personality also means the consistent pattern of emotion, behavior, cognition and desire (goal) that relatively last long, irrelevant of time and space (Revelle and Scherer, 2009). Ryckman (2000) regarded personality is a series of dynamic and organized characteristics owned by an individual, and said these characteristics uniquely influence individual's perception, motive and behavior in various situations.

Meanwhile, emotion is explained by various expressions including nervous system's autonomic activation, subjective experience, facial expression, specific behavior or predisposition belonging to social roles (Wells and Matthews, 1994). Emotion is complex interaction of subjective and objective factors, and unfolds through the intervention of nervous and hormone systems. Emotion may cause affective experience like awakening or pleasantness/unpleasantness, may induce cognitive process like emotion-related perception, calmness and classification, or operate extensive physiological control on provocative conditions. And, emotion is expressive, goal-oriented and induces active behavior, despite not necessarily so (Kleinginna and Kleinginna, 1981).

If emotion is expressed as the integration of feeling, behavior, evaluation and desire in specific time and space, personality is the integration of these factors, beyond time and space (Ortony et al., 2005). Many studies on the relationship between emotion and personality have been carried out. Plutchik (1980) proposed that trait (personality characteristics), which is relatively perpetual and reflects individual's uniqueness, can be derived from emotion or the result deriving from emotional combination and that people tend to judge other person's personality trait on the basis of observable emotional responses.

Spielberger et al. (1983) applied the concept of predisposition to personality, and defined and conceptualized the predisposition experiencing specific emotion, irrelevant of time and space, as dispositional emotion. Lerner and Kletner (2001) researched dispositional terror and anger, and Watson and Clark (1984) established the concept of dispositional negative emotionality. In addition, studies on the relationships between personality characteristics and anger (Spielberger et al., 1983; Martin et al., 1999) between personality characteristics and happiness (Klein, and Kunda, 1992; Klein, 1993; Lu, 1995, Myers and Diener, 1995; Diener, Suh, Lucas, and Smith, 1999), and the relationship between stress assessment and handling (McCrae and Costa, 1986; Holahan and Moos, 1987; Henderson, 1981, Gunthert, Cohen, and Armeli, 1999; Lazarus and Folkman, 1984) have been conducted. In Korea, the studies on the relationship between the difference in sadness and anger as dispositional emotion (Han et al., 2003), relationship between personality characteristics and emotional behavior expressions (Min et al., 1998) and the relationship of behaviors to cope with stress (Kim, 1985; Kim, 2003) have been performed.

Most studies on personality and emotional responses, however, have targeted adults, and the studies targeting children are limited. Childhood is a very important period for personality formation, and is a critical period to control and learn the process of expressing one's own emotional state. For this reason, there is a need to undertake studies on the personality and emotion of children. Some researchers carried out research on the personality and emotion of children, but they mainly measured personality development or emotional state individually (Koo et al., 2006; Tagiuri, 1969; Wellman, 1990; Lee, 2004; Kim, 2005). Also, some studies identifying the relationship between personality and emotion reported the relationship between personality and a single emotion like the relationship between personality characteristics and anger (Kim and Hong, 2008; Nam, 2010) or the relationship between personality type and happiness (Lee, 1986; Kim, 2008). However, these studies limited research area to the extent that emotional side effects, due to experimental manipulation, are small in the case of inducing positive or negative emotion.

This study aims to check the relationship between personality characteristics and psychological responses on basic emotions such as happiness, sadness, anger, stress and boring, not limited to a single emotion. To this end, this study selected children aged 7 to 9 , who were in the period of early stage of schooling. Children in this period demonstrate socialized and various emotional expressions, as they are developed cognitively, as they approach specific manipulation period, according to Piajet (1970). To 
induce proper emotions for the children, this study shaped the experiment setting for them to have emotionally inductive context and have sufficient emotional experience, and restored their psychological stability state not to leave the side effects of emotional experience for the children. This study actually verified emotion protocols for children as primary verification as a subjective measuring method, and analyzed detailed responses by each emotion using an objective measuring method later.

\section{Method}

\subsection{Subjects}

We conducted Korean PIC (Personality Inventory for Children) targeting 94 boys and girls aged 7 to 9 ( 47 boys and 47 girls), who were the kindergarteners and the first and second graders in one kindergarten and four elementary schools located in Daejeon City. They were selected under the consent of their home room teachers and parents. By sending the K-PIC test sheets to their homes, their parents answered the test sheet. The test sheets with finished answers were supposed to be sent back to our lab using the enclosed return envelope. Among the returned test sheets, 64 children were selected and participated in this study's experiment, except for the test sheets with omitted answers to the questions and the insincere answered-test sheets.

\subsection{The personality inventory for children (PIC)}

The K-PIC (Korean Personality Inventory for Children) (Sohn, Chi and Kim, 1994) was used to measure childrens' personality. The K-PIC is the scale rated as having high reliability and validity through preceding studies (Jeon, 1995). PIC is a personality test produced and standardized by Wirt, Seat and Broen (1977), and has been used to assess and diagnose child's personality for a long time. The PIC can measure comprehensive personality of children aged 3 to 16 and adolescent's behavior, emotion, perception and family relation through parents' responses. Therefore, the PIC supplements a demerit that other personality tests have, namely, a demerit that is inappropriate to children, who have lacking language understanding and expressing abilities, by using a selfreporting mode.

The sub-scales of the PIC used in this study are as follows: 1) Family relation: FAM, 2) Hyperactivity: HPR, 3) Internalization: INT, 4) Development: DVL, 5) Defensiveness: DEF, 6) Lie: L, 7) Anxiety: ANX, 8) Delinquency: DLQ, 9) Social Skill Deficits: SSK, 10) Social Skills: SOC, 11) Achievement: ACH, 12) Somatic Concern: SOM, 13) Depression: D, 14) Cognitive Development: COG, 15) Frequency: F, 16) Adjustment: ADJ, 17) Psychosis: PSY, 18) Intellectual Screening: IS, 19) Withdrawal: WDL, 20) Nurture: N.

\subsection{Emotional stimuli}

We induced five types of emotions (happiness, sadness, anger, stress and boring) using Child Emotion Induction Protocol (Jang et al., 2009). The Emotion Protocol consists of the combination of story, doll, color and lighting that generate music and imagery familiar to children, and they were presented to children for maximum two minutes (Table 1). Figure 1 shows an example of emotion stimuli to induce sadness.

\subsection{Emotion assessment scale}

To assess the emotions that children experience, we used Emotion Assessment Scale for Children (Jang et al., 2009). This scale consists of a stage to categorize emotions that a child experiences into specific emotion names, and a stage to quantify emotion intensity in the mode of scoring of the experienced emotion intensity. The stage to categorize emotion names is to find out the suitability of the protocol, and the stage to quantify emotion intensity is to verify protocol's effectiveness. The suitability of the protocol means conformity degree between experimenter-intended emotions and those that children actually experienced. The 
Table 1. The standardized emotion induction protocols

\begin{tabular}{|c|c|c|c|c|c|}
\hline & HAP & SAD & ANG & STR & $\mathrm{BOR}$ \\
\hline Music & $\begin{array}{l}\text { Theme song of 'Pokémon' } \\
\text { and 'Dooly' }\end{array}$ & 'Romance' by Yuhki Kuramoto & None & None & None \\
\hline Lighting & Rotation of red, blue and green & Blue & Red & Blinking white & Green \\
\hline Story & Praise & Sympathy & False charge & Nitpicking & None \\
\hline Doll & Pooh & Eeyore & Bulldog & Daisy Duck & None \\
\hline
\end{tabular}

Abbreviation: HAP - happiness, SAD - sadness, ANG - anger, STR - stress, BOR - boring

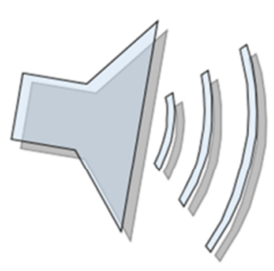

MUSIC

['Romance' by Yuhki Kuramoto]
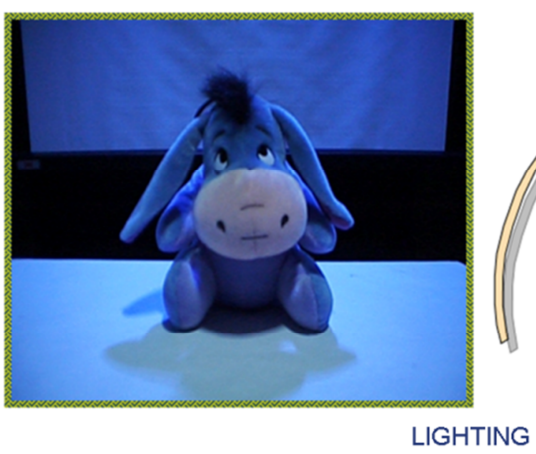

TOY STORY

[Sympathy Induction]

[Blue]

Figure 1. The example of emotional stimuli

effectiveness of the protocol means the intensities of emotions that children actually experienced. For suitability of the protocol, children reported the emotions (sadness, happiness, anger, etc.) that they experienced in the stage to categorize emotions, and the suitability was analyzed with the number of the cases conforming to the experimenter-intended emotions. The effectiveness of the protocol assessed the emotion intensity experienced by children using Five-Point Likert Scale, and the star-scoring type was presented to help children's understanding. Namely, when a child experienced emotion a little, one star was marked, and five stars were marked for the emotion that was experienced the most by a child. At the stage to categorize emotions, when a child reported he/she did not experience any emotion, the effectiveness was not assessed.

\subsection{Experimental environment}

We set the room with friendly environment to help child's natural emotion induction. In the $12 \times 6 \mathrm{~m}$ (width $\mathrm{x}$ length) soundproof room, where noise was interrupted (noise: $35 \mathrm{~dB}$ and less), color stimulation was presented with lighting, and music and story were presented through speakers. A CCTVE was installed in the central front of the room to observe children's unexpected situations and behaviors during the experiment. In the outside of the room, a computer and an audio system were installed to present stimuli to the children, and also TV and video camera equipment were installed to check and store the inputted information through CCTV. Figure 2 shows the experimental environment, where the experiment was carried out.

When a child enters the room, the child has stabilization time to adjust to the experimental environment and forms a rapport with the experimenter. After the experimenter checks child's emotional expressivity, conducts emotion discrimination training, and lets the child be familiar with the experiment method. When the experiment starts, the child has two minutes of stabilization time, and 


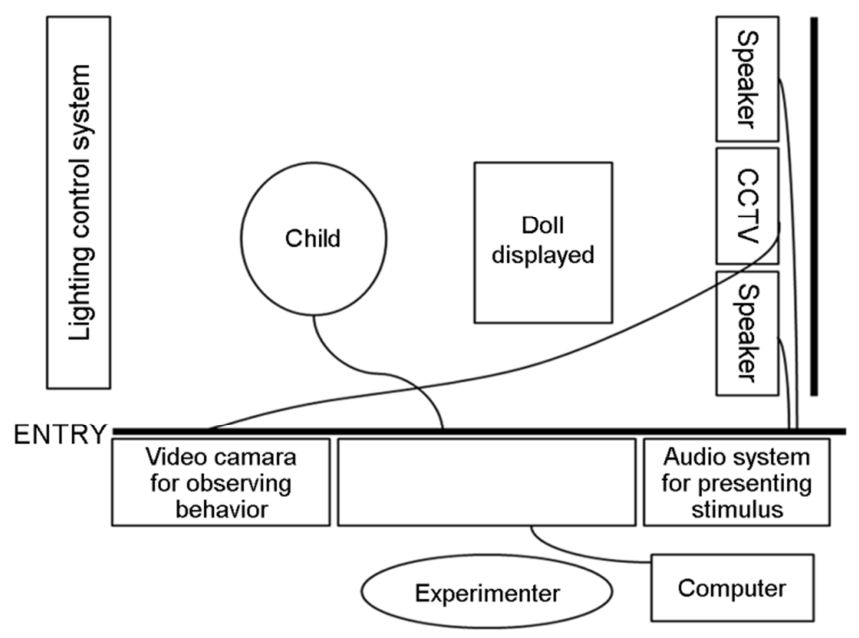

Figure 2. Experimental environment

receives emotional stimuli for about two minutes. After the emotion stimuli are presented, the experimenter enters the room, and checks the child's emotional state. After the child experienced five types of emotions, the experiment is finished, and the experimenter makes the child stable emotionally. The emotion stimuli are randomly presented to the child, and the total time for the experiment is about 40 minutes.

\section{Results}

This study presented total 64 children's psychological responses, namely, the study results were divided into emotion protocol's suitability, effectiveness and child's emotion responses according to personality characteristics.

\subsection{Suitability and effectiveness of emotion protocols}

Based on the total 64 children's emotion assessment scale, we analyzed the suitability and effectiveness by five emotion protocols using SPSS 16.0 Statistical Program. To check the suitability of the emotion protocols, we conducted frequency analysis using the rating value of the questions categorizing the emotion names. That is, the number of cases matching the experimenter-intended emotions and the children-reported emotions was indicated as percentage. For example, when 32 children out of 64 children reported sadness as the emotion they experienced regarding sadness emotion protocol, the suitability of the sadness protocol is $50 \%$. As a result of suitability for each emotion, happiness was $98.3 \%$, sadness $84.7 \%$, anger $80.7 \%$, stress $77.2 \%$ and boring $91.2 \%$, respectively, and they reached target emotions. The effectiveness of emotion protocols was analyzed with technical statistical values by each emotion using quantified emotion intensity through five-point scale. In other words, the emotion intensity scores evaluated by children were averaged and presented as percentage. For instance, when average intensity of anger emotion experienced by children was 4.0 , the effectiveness of the anger protocol is $80 \%$. As a result of protocol's effectiveness analysis, happiness was $93.8 \%$, sadness $81.2 \%$, anger $90.5 \%$, stress $86.6 \%$ and boring $80.4 \%$. This means that each emotion protocol sufficiently reflected target emotions and children properly and effectively experienced target emotions (Table 2).

\subsection{Differences of emotional responses by personalities}

We compared children's psychological responses by dividing the responses to each emotion according to each personality 
Table 2. The suitability and effectiveness of emotion protocols

\begin{tabular}{l|l|l|l|l|l|l|}
\hline & HAP & SAD & ANG & STR & BOR & M \\
\hline Suitability & 98.3 & 84.7 & 80.7 & 77.2 & 91.2 & 86.4 \\
\hline Effectiveness & 93.8 & 81.2 & 90.5 & 86.6 & 80.4 & 86.5 \\
\hline
\end{tabular}

characteristics. Among 20 detailed personality scales composing the PIC, we analyzed psychological responses by selecting 27 children corresponding to upper $25 \%$ and lower $25 \%$ of each personality scale out of the entire experiment participating children, based on 16 sub-scale scores, except lie, frequency, defensiveness and nurture scales. To analyze psychological responses of the two groups according to personality, we performed a cross analysis and an independent group $t$-test. The cross analysis is to test ratio differences of categorization type variables, and was used to verify the differences of suitability between the two groups. The independent group $t$-test is a parametric test technique used to decide whether two groups' averages have significant differences. The t-test was analyzed to verify the differences of effectiveness between the two groups.

The suitability of emotion protocols is shown in Table 3. Concerning the suitability by emotion between the groups having high and low scores in each scale, hyperactivity (HPR) and adjustment (ADJ) scales showed significant differences, and family relation (FAM) also showed a similar trend. The significant difference between the groups having high and low scores of HPR was found only in sadness condition $\left(\chi^{2}=3.94, p<.05\right)$. Namely, the ratio of the group having high HPR score that reported sadness was higher than that of the group having low HPR score. Significant difference of emotion responses between the groups having high and low scores in ADJ scale was shown only in stress condition $\left(\chi^{2}=4.79, p<.05\right)$. The group having high score in $A D J$ scale had lower ratio of stress reporting than the group having low score in ADJ scale. The suitability of the group having high score in FAM scale was lower than the group having low score in FAM scale $\left(\chi^{2}=3.94, p=.06\right)$.

Table 3. The suitability of each emotion protocol according to high- and low-level of personality scales score

\begin{tabular}{|c|c|c|c|c|c|c|}
\hline & & HAP & SAD & ANG & STR & BOR \\
\hline \multirow{2}{*}{ FAM } & High & 92.9 & 81.8 & 71.4 & 30.8 & 85.7 \\
\hline & Low & 100.0 & 78.6 & 71.0 & 70.0 & 100.0 \\
\hline \multirow{2}{*}{ HPR } & High & 93.3 & 94.1 & 73.3 & 60.0 & 84.6 \\
\hline & Low & 100.0 & 66.7 & 75.0 & 50.0 & 94.1 \\
\hline \multirow{2}{*}{ INT } & High & 100.0 & 91.7 & 66.7 & 72.7 & 83.8 \\
\hline & Low & 91.7 & 91.7 & 90.9 & 63.6 & 90.9 \\
\hline \multirow{2}{*}{ DVL } & High & 100.0 & 84.6 & 69.2 & 46.2 & 91.7 \\
\hline & Low & 100.0 & 70.0 & 66.7 & 77.8 & 90.0 \\
\hline \multirow{2}{*}{ ANX } & High & 100.0 & 90.9 & 90.0 & 60.0 & 100.0 \\
\hline & Low & 100.0 & 84.6 & 75.0 & 66.7 & 100.0 \\
\hline \multirow{2}{*}{ DLQ } & High & 92.9 & 71.4 & 71.4 & 42.9 & 76.9 \\
\hline & Low & 100.0 & 78.9 & 61.1 & 55.6 & 94.7 \\
\hline \multirow{2}{*}{ SSK } & High & 100.0 & 81.3 & 80.0 & 60.0 & 87.5 \\
\hline & Low & 100.0 & 86.7 & 80.0 & 60.0 & 93.3 \\
\hline
\end{tabular}


Table 3. The suitability of each emotion protocol according to high- and low-level of personality scales score (Continued)

\begin{tabular}{|c|c|c|c|c|c|c|}
\hline & & HAP & SAD & ANG & STR & BOR \\
\hline \multirow{2}{*}{ SOC } & High & 92.3 & 76.9 & 58.3 & 46.2 & 75.0 \\
\hline & Low & 100.0 & 83.3 & 75.0 & 58.3 & 100.0 \\
\hline \multirow{2}{*}{$\mathrm{ACH}$} & High & 94.1 & 82.4 & 58.8 & 58.8 & 86.7 \\
\hline & Low & 100.0 & 87.5 & 73.3 & 60.0 & 87.5 \\
\hline \multirow{2}{*}{ SOM } & High & 90.0 & 90.0 & 54.5 & 40.0 & 80.0 \\
\hline & Low & 100.0 & 83.3 & 72.7 & 54.5 & 91.7 \\
\hline \multirow{2}{*}{$D$} & High & 92.3 & 76.9 & 76.9 & 50.0 & 84.6 \\
\hline & Low & 100.0 & 73.3 & 75.0 & 66.7 & 100.0 \\
\hline \multirow{2}{*}{ COG } & High & 100.0 & 85.7 & 64.3 & 42.9 & 91.7 \\
\hline & Low & 100.0 & 85.7 & 76.9 & 69.2 & 100.0 \\
\hline \multirow{2}{*}{ ADJ } & High & 91.7 & 83.3 & 72.7 & 33.3 & 81.8 \\
\hline & Low & 100.0 & 90.9 & 80.0 & 80.0 & 100.0 \\
\hline \multirow{2}{*}{ PSY } & High & 91.7 & 75.0 & 66.7 & 69.2 & 83.3 \\
\hline & Low & 100.0 & 78.6 & 76.9 & 41.7 & 100.0 \\
\hline \multirow{2}{*}{ IS } & High & 100.0 & 88.9 & 66.7 & 33.3 & 85.7 \\
\hline & Low & 88.9 & 66.7 & 55.6 & 55.6 & 77.8 \\
\hline \multirow{2}{*}{ WDL } & High & 91.7 & 75.0 & 54.5 & 50.0 & 75.0 \\
\hline & Low & 100.0 & 68.8 & 75.0 & 50.0 & 93.8 \\
\hline
\end{tabular}

Table 4 shows the result of the effectiveness of emotion protocols. Concerning the effectiveness by emotion between the groups having high and low scores of each scale, significant differences were shown in only psychosis (PSY) scale and intellectual screening (IS) scale. Significant difference between the groups having high and low PSY scale scores was demonstrated in the anger emotion condition ( $t=2.21, p<.05$ ). The group having the predisposition of PSY experienced anger more strongly that the group having low predisposition of PSY. In intellectual screening (IS) scale, significant difference between the groups having high and low IS scale scores was demonstrated in sadness condition $(t=2.66, p<.05)$. Children in the group having low score in IS scale felt sadness stronger than the children in the group having low score in IS scale.

Table 4. The effectiveness of each emotion protocol according to high- and low-level of personality scales score

\begin{tabular}{l|l|c|c|c|c|c}
\hline \multicolumn{2}{l|}{} & HAP & SAD & ANG & STR & BOR \\
\hline \multirow{3}{*}{ FAM } & High & $95.4 \pm 3.3$ & $87.3 \pm 4.9$ & $90.0 \pm 6.1$ & $91.4 \pm 4.0$ & $81.8 \pm 6.9$ \\
\cline { 2 - 7 } & Low & $87.3 \pm 6.2$ & $75.6 \pm 7.3$ & $95.0 \pm 3.3$ & $92.5 \pm 3.7$ & $81.8 \pm 5.0$ \\
\hline \multirow{3}{*}{ HPR } & High & $94.3 \pm 3.3$ & $80.0 \pm 4.2$ & $96.7 \pm 2.2$ & $89.1 \pm 3.1$ & $72.7 \pm 6.8$ \\
\cline { 2 - 7 } & Low & $91.8 \pm 3.9$ & $83.8 \pm 4.2$ & $95.4 \pm 2.4$ & $90.8 \pm 3.7$ & $86.7 \pm 4.2$ \\
\hline
\end{tabular}


Table 4. The effectiveness of each emotion protocol according to high- and low-level of personality scales score (Continued)

\begin{tabular}{|c|c|c|c|c|c|c|}
\hline & & HAP & SAD & ANG & STR & BOR \\
\hline \multirow{2}{*}{ INT } & High & $91.7 \pm 3.9$ & $83.6 \pm 5.9$ & $90.0 \pm 7.6$ & $92.5 \pm 3.7$ & $78.0 \pm 7.0$ \\
\hline & Low & $89.1 \pm 5.6$ & $76.4 \pm 4.5$ & $90.0 \pm 3.3$ & $87.5 \pm 5.3$ & $82.0 \pm 3.6$ \\
\hline \multirow{2}{*}{ DVL } & High & $87.7 \pm 4.8$ & $83.6 \pm 5.3$ & $81.8 \pm 8.3$ & $83.0 \pm 9.2$ & $85.5 \pm 4.7$ \\
\hline & Low & $92.0 \pm 4.4$ & $83.3 \pm 3.3$ & $100 \pm 0$ & $91.4 \pm 5.9$ & $82.2 \pm 6.2$ \\
\hline \multirow{2}{*}{ ANX } & High & $87.3 \pm 5.6$ & $78.0 \pm 4.7$ & $88.9 \pm 6.8$ & $88.6 \pm 4.0$ & $80.0 \pm 7.1$ \\
\hline & Low & $95.4 \pm 2.4$ & $83.6 \pm 5.3$ & $88.0 \pm 6.1$ & $84.4 \pm 4.4$ & $78.5 \pm 6.6$ \\
\hline \multirow{2}{*}{ DLQ } & High & $96.9 \pm 2.1$ & $90.0 \pm 4.5$ & $90.0 \pm 6.1$ & $84.0 \pm 5.0$ & $74.0 \pm 7.3$ \\
\hline & Low & $91.6 \pm 3.8$ & $78.6 \pm 4.4$ & $81.7 \pm 6.3$ & $87.3 \pm 3.0$ & $75.0 \pm 5.6$ \\
\hline \multirow{2}{*}{ SSK } & High & $91.3 \pm 4.1$ & $84.6 \pm 4.0$ & $95.4 \pm 2.4$ & $77.5 \pm 7.4$ & $83.1 \pm 6.3$ \\
\hline & Low & $96.0 \pm 2.1$ & $81.5 \pm 5.3$ & $87.7 \pm 4.8$ & $87.3 \pm 4.1$ & $78.5 \pm 5.3$ \\
\hline \multirow{2}{*}{ SOC } & High & $93.3 \pm 2.8$ & $82.2 \pm 5.2$ & $100 \pm 0$ & $87.5 \pm 5.3$ & $84.4 \pm 8.7$ \\
\hline & Low & $93.3 \pm 3.8$ & $84.0 \pm 5.8$ & $86.7 \pm 6.7$ & $88.9 \pm 4.8$ & $80.0 \pm 5.5$ \\
\hline \multirow{2}{*}{$\mathrm{ACH}$} & High & $95.3 \pm 2.7$ & $76.0 \pm 4.5$ & $92.3 \pm 4.8$ & $91.4 \pm 2.7$ & $84.3 \pm 6.4$ \\
\hline & Low & $95.0 \pm 2.9$ & $84.3 \pm 3.1$ & $95 \pm 2.6$ & $87.7 \pm 4.3$ & $81.5 \pm 5.8$ \\
\hline \multirow{2}{*}{ SOM } & High & $90.0 \pm 4.5$ & $78.0 \pm 7.6$ & $91.4 \pm 4$ & $82.9 \pm 5.2$ & $77.5 \pm 9.6$ \\
\hline & Low & $98.3 \pm 1.7$ & $76.0 \pm 5.8$ & $92.5 \pm 3.7$ & $82.9 \pm 5.2$ & $76.4 \pm 4.5$ \\
\hline \multirow{2}{*}{$D$} & High & $93.3 \pm 3.8$ & $86.0 \pm 4.3$ & $98 \pm 2.0$ & $87.5 \pm 3.7$ & $78.2 \pm 6.9$ \\
\hline & Low & $96.7 \pm 2.2$ & $84.0 \pm 5.8$ & $88.3 \pm 5.2$ & $87.3 \pm 4.1$ & $86.0 \pm 3.1$ \\
\hline \multirow{2}{*}{ COG } & High & $90.0 \pm 4.6$ & $76.7 \pm 5.9$ & $91.7 \pm 5.2$ & $81.1 \pm 9.5$ & $83.6 \pm 4.5$ \\
\hline & Low & $92.9 \pm 3.4$ & $80.0 \pm 4.3$ & $94.0 \pm 3.1$ & $90.0 \pm 3.3$ & $76.7 \pm 4.8$ \\
\hline \multirow{2}{*}{ ADJ } & High & $93.3 \pm 3.8$ & $81.8 \pm 3.3$ & $97.8 \pm 2.2$ & $93.3 \pm 3.3$ & $82.0 \pm 8.1$ \\
\hline & Low & $98.2 \pm 1.8$ & $81.8 \pm 1.8$ & $98.0 \pm 2.0$ & $96.0 \pm 2.7$ & $92.0 \pm 3.3$ \\
\hline \multirow{2}{*}{ PSY } & High & $94.5 \pm 2.8$ & $90.0 \pm 5.3$ & $100 \pm 0$ & $88.6 \pm 4.0$ & $86.0 \pm 7.9$ \\
\hline & Low & $94.3 \pm 3.3$ & $80.0 \pm 5.4$ & $86.0 \pm 6.0$ & $88.0 \pm 4.4$ & $77.1 \pm 4.6$ \\
\hline \multirow{2}{*}{ IS } & High & $97.8 \pm 2.2$ & $67.5 \pm 6.5$ & $97.1 \pm 2.9$ & $92.0 \pm 4.9$ & $86.7 \pm 6.7$ \\
\hline & Low & $95.6 \pm 4.4$ & $88.6 \pm 4.0$ & $96.7 \pm 3.3$ & $91.4 \pm 5.9$ & $77.5 \pm 8.8$ \\
\hline \multirow{2}{*}{ WDL } & High & $92.7 \pm 4.1$ & $75.6 \pm 6.5$ & $96.7 \pm 3.3$ & $88.0 \pm 4.9$ & $86.7 \pm 4.7$ \\
\hline & Low & $96.3 \pm 2.7$ & $83.6 \pm 5.3$ & $89.2 \pm 4.9$ & $85.0 \pm 3.6$ & $78.6 \pm 4.4$ \\
\hline
\end{tabular}

\section{Conclusion}

As a result of this study, the emotion response differences on emotion protocols by the two groups (upper $25 \%$ and lower $25 \%$ groups) were demonstrated significantly in only sadness and stress conditions, according to the groups having high and low scores in ADJ scale and HPR scale. According to Jeon (1995), ADJ and HPR scales belong to the factor I scale, namely, nurture/ self-control deficits, out of four wide factors in the PIC: the four wide factors in PIC are as follows: factor I scale - nurture/self- 
control deficit, factor II scale - lack of social skills, factor II scales - internalization, somatic concern, factor IV scales -cognitive development. They also reflect anger causing impulsiveness and problems, inappropriateness of friend relation, lacking moral development and lack of activity at school, and has greatly related with depression scale and family relation scale.

HPR scale is the scale measuring delinquent child's behavioral characteristics. The ratio reporting sadness in sadness condition of the children having high score in HPR scale was higher than the children having low HPR scale score. HPR scale is the scale to discriminate children showing the characteristics related with hyper exercise syndrome (appropriate to diagnose attention deficit disorder accompanied by past activity, based on DSM-III). High increase in this scale implies excessively bragging, distraction and no good attitude in classes, lack of patience on frustration and problem in human relations. Extreme increase in HPR scale means taking problematic behavior not controlling antagonism, and not conforming to sanctions at home or at school. Namely, it was confirmed that children with high score in HPR more precisely perceived sadness emotion than normal children.

ADJ scale is a general scale on psychological state not adjusting well. The children with high score in ADJ scale are highly related with non-adjustment degree such as behavioral disorder, depression, lack of patience, delinquency predisposition, mood disorder and no smooth human relations. According to preceding studies, the disorder degree of the children having low score in ADJ scale is relatively weaker than those receiving high score in ADJ scale (Seat, 1969). According to the result of this study, the stress-reporting ratio of the group with high score in ADJ scale was lower than the group receiving high score in ADJ scale. Namely, this means that the children that cannot adjust well do not recognize their-experienced stress condition as stress.

This study demonstrated the suitability of the group with high score in FAM scale was lower than the group with low score during the stress condition, despite not statistically significant. The FAM scale is to measure efficient relation with family and identify the influences of the characteristics of family and parents on child's mental pathology. As the FAM scale score is higher, it implies the instability of parents' marriage life, parents' emotional conflict or alcoholic drinking or drug addiction problems. The extremely high score means that the relation between parents and child is bad or punishment-centered relation. The study result is that emotion responses of the children with unstable family relation on the stress condition were not precise, compared to the children with more stable family relation. In summary, because the children with a problem of nurturing or self-control deficit have such problems as distraction, impulsiveness and hyperaction, they get more stress by the surroundings, and are known to feel strong sadness frequently. However, it is identified that they do not precisely recognize stress in the stress situation, compared to the normal children through this study.

Meanwhile, in emotion experience intensity on emotion protocols, significant differences were shown between the two groups (upper and lower 25\%) in psychosis (PSY) and intellectual screening (IS) scales. PSY scale is to discriminate children showing psychiatric symptom. High score in this scale reflects social shrinking and isolation, social adjustment skills deficit, delay in acquisition of skills necessary for everyday life, depression, in appropriate emotion expression and cognitive deficit. The high score means emotional instability, social skills deficit and social isolation. The group receiving high score in PSY scale experienced anger more strongly than the group receiving low score. Anger damages psychological and mental functions and induces antisocial behaviors. The result in this study supports the experience of anger is related to PSY scale measuring psychological and mental function damages.

In the experienced emotion intensity, difference was demonstrated between the two groups in IS scale. This consists of the sub-questions including cognition deficit, division from psychiatric symptom, cooperation and adjustment deficits, low academic capability, emotional disorder, colleague relation, delay in language development, recognition on insufficient potential and cognitive disorder. The group receiving high score in IS scale reported they felt less sadness than the group receiving low score. This can be explained by the research of Dauber and Benbow (1996) saying that highly intelligent children are more emotionally sensitive than the children who are not highly intelligent. Namely, as children's intellectual ability is higher, they are more sensitive 
emotionally, and thus, they feel sadness more strongly.

In conclusion, it was confirmed that hyperaction, nurture issue like adjustment, and family relation, psychiatric symptom and intellectual ability affect the accuracy and intensity of emotion responses. This study is meaningful in that it offers guidelines on the study of children's emotion that has not been properly addressed, due to methodological constraints. However, this study has limitation in the analysis process. Because the study subjects were children, more than $70 \%$ of each personality scale score was distributed in the center of normal distribution curve. Also, t-test was carried out using the data corresponding to the upper and lower $25 \%$ groups in each personality scale score, since the purpose of this study is to check differences in emotion responses according to high and low level of personality characteristics. This study performed 85 sessions of t-test for 16 personality subscales and five emotion variables and therefore, control on the increase of one type of error was not conducted. If one type of error increases, the probability of randomly significant result is high. To solve this problem, further study needs to analyze by searching for another statistical technique that can minimize statistical errors like regression analysis, in which five emotions are set as dependent variables and each detailed area of personality is set as a prediction variable.

\section{Acknowledgements}

This paper was carried out through assistance of the National Research Foundation of Korea (No. NRF-2006-2005087) with the finances of the government (Ministry of Science, ICT and Future Planning) in 2013.

\section{References}

Bueger, J.M. and Caldwell, D.F., Personality, social activities, job-search behavior and interview success: Distinguishing between PANAS trait positive affect and NEO extraversion, Motivation and Emotion, 24(1), 51-62, 2000.

Chiles, J.A., Miller, M.L. and Cox, G.B., Depression in an adolescent delinquent population, Archives of General Psychiatry, 37(10), $1179-1184,1980$.

Dauber, S.L. and Benbow, C.P., Aspects of personality and peer relations of extremely talented adolescents, Gifted Child Quarterly, 34, 10-15, 1990.

Diener, E., Suh, E.M., Lucas, R.E. and Smith, H.L., Subjective well-being, Psychological Science in the Public Interest, 5(2), 1-31, 1999.

Gunthert, K.C., Cohen, L.H. and Armeli, S., The role of neuroticism in daily stress and coping, Journal of Personality Social Psychology, 77(5), 1087-1100, 1999.

Han, S.H., Cognitive and personality characteristics of sadness-prone and anger-prone people, Seoul National University, Seoul, Republic of Korea, 2003.

Henderson, A.S., Social relationships, adversity and neurosis: An analysis of prospective observations, British Journal of Psychiatry, 138, 391-398, 1981.

Holahan, C.J. and Moos, R.H., Social support and psychological distress: A longitudinal analysis, Journal of Abnormal Psychology, 90, 365-370, 1987.

Jang, E.H., Yang, G.H., Lee, J.M., Chung, M.A. and Sohn, J.H., Development of protocol for standardized emotion induction in 
children, Korean Journal of the Science of Emotion and Sensibility, 12(4), 381-392, 2009.

Jeon, B.G., Standardization for Korean version of Personality Inventory for Children, Chungnam National University, Daejeon, Republic of Korea, 1995.

Kim, C.D., Depression and ways of stress coping, Seoul National University, Seoul, Republic of Korea, 1985.

Kim, C.S., The effects of personality trait dimension on stress response in adolescents, Korean Journal of Youth Studies, 10(4), $21-35,2003$

Kim, T.S. and Hong, S.H., Mediating effects of trait anger and cognitive appraisal resource on the relationship between children's anger-inducing event and anger-out, Korean Journal of School Psychology, 5(3), 269-287, 2008.

Kim, Y.H., The relationships among children's happiness, personality types and parent-child communication patterns, Seoul Womans University, Seoul, Republic of Korea, 2008.

Kim, Y.H., The comparison analysis for emotional characteristics between attention deficit and normal youth, Ajou University, Suwon, Republic of Korea, 2005.

Klein, P., The handbook of psychological testing, London: Routledge, 1993.

Klein, W.M. and Kunda, Z., Motivated person perception: Constructing justifications for desired beliefs, Journal of Experimental Social Psychology, 28, 145-160, 1992.

Kleinginna, P.R. and Kleinginna, A.M., A categorized list of motivation definitions with a suggestion for a consensual definition, Motivation and Emotion, 5, 263-291, 1981.

Koo, J.S., Ghim, H.R., Kim, K.M., Yang, H.Y., Ko, S.N. and Chung, M.S., Children's understanding of personality traits: with focus on trait inference and situational and temporal stability, Korean journal of developmental psychology, 19(4), 1-20, 2006.

Kovacs, M., The Children's Depression Inventory: A self-rated depression scale for school-aged youngsters, Unpublished manuscript. University of Pittsburgh, USA, 1983.

Krakowski, A.J., Depressive reactions of childhood depression: Developmental considerations, In S. R. Shirk (ED.), Cognitive development and child psychotherapy (pp. 187-204), New York: Plenum, 1970.

Lazarus, R.S. and Folkman, S., Stress, appraisal, and coping, New York: McGraw-Hill, 1984

Lee, S., A study on the development of child's attitude to sadness, Ewha Woman University, Seoul, Republic of Korea, 1986.

Lee, T.H., Relationship of depression, irrational faith and the school adaptation of elementary school students, Chuncheon National University of Education, Chuncheon, Republic of Korea, 2004.

Lerner, J.S. and Keltner, D., Fear, anger, and risk, Journal of Personality and Social Psychology, 81(1), 146-159, 2001. 
Lu, L., The relationship between subjective well-being and psychosocial variables in Taiwan, Journal of Social Psychology, 135, 351-357, 1995.

Martin, R., Wan, C.K., David, J.P., Wegner, E.L., Olson, B.D. and Watson, D., Style of anger expression: Relation to expressivity, personality, and health, Personality and Social Psychol Bulletin, 25, 1196-1207, 1999.

McCrae, R.R. and Costa, P.T. Jr., Personality, coping and coping effectiveness in an adult sample, Journal of Personality, 54, 385405, 1986.

Min, K.H., Kim, J.H., Hwang, S.H. and Jang, S.M., Variations in emotion response patterning across genders, generations, and personality types, Korean Journal of Social and Personality Psychology, 12(2), 119-140, 1998.

Myers, D. and Diener, E., Who is happy?, Psychological Science, 6, 10-19, 1995.

Nam, J.H., The relationship between children's personality traits and anger expression: The mediating effect of trait anger, Hanyang University, Seoul, Republic of Korea, 2010.

Ortony, A., Norman, D. and Revelle, W., Effective functioning: $A$ three level model of affect, motivation, cognition, and behavior, In J. Fellous and M. Arbib (Eds.), Who needs emotions? The brain meets the machine. New York: Oxford University Press, 2005.

Piaget, J., Piaget's theory, P. H. Mussen (Ed.), Charmichael's manual of child psychology (3rd ed., Vol. 1). New York: Wiley, 1970.

Plutchik, R., Emotion: A psychoevolutionary synthesis, New York: Harper and Row, 1980.

Puig-Antich, J., Major depression and conduct disorder in prepuberty, Journal of the American Academy of Child Psychiatry, 21(2), 118-128, 1982

Revelle, W. and Scherer, K.R., Personality and emotion, In D. Sander and K. R. Scherer (Eds.), The Oxford companion to emotion and the affective sciences. Oxford: Oxford University Press, 2009.

Ryckman, R.M., Theories of personality, CA: Wadsworth Pub. Co., 2000.

Sohn, J.H., Chi, Y.N. and Kim, S.H., A pilot study for Korean version of personality inventory for children, Sungkok Review, 25, 425-458, 1994.

Spielberger, C.D., Gorusch, R.L., Lushene, R.E., Vagg, P.R. and Jacobs, G.A., Manual for the state-trait anxiety inventory (Form Y): Self-evaluation questionnaire, Palo Alto, CA: Consulting Psychologists, 1983.

Tagiuri, R., Person perception, In G. Lindzey and E. Aronson (Eds.), The handbook of social psychology (vol. 3). Reading, MA: Addison-Wesley, 1969.

Watson, D. and Clark, L.A., Negative affectivity: The disposition to experience aversive emotional stress, Psychological Bulletin, 96, 465-490, 1984.

Wellman, H.M., The child's theory of mind, Cambridge, MA: Bradford, 1990. 
Wells, A. and Matthews, G., Attention and emotion, Hillsdale: LEA, 1994.

Wirt, R.D., Seat, P.D. and Broen, W.E., The personality inventory for children, Los Angeles: Western Psychological Services, 1977.

\section{Author listings}

Eun Hye Jang: cleta4u@etri.re.kr

Highest degree: PhD, Department of Psychology, Chungnam National University

Position title: Senior Member of Engineering Staff, BT Convergence Technology Research Department, Electronics and Telecommunications Research Institute

Areas of interest: Emotion Recognition, Psychophysiology, Cognition Convergence

Youngji Eum: youngjieum@gmail.com

Highest degree: MA, Department of Psychology, Chungnam National University

Position title: PhD student, Department of Psychology, Chungnam National University

Areas of interest: Emotion, Cognition, Neuroscience, Psychophysiology

Suk-Hee Kim: sookheekim22@gmail.com

Highest degree: PhD, Department of psychology, the University of Korea

Position title: Professor, Department of Professional Counseling \& Psychotherapy, Wonkwang University

Areas of interest: Counseling, Psychotherapy

Jin-Hun Sohn: jhsohn@cnu.ac.kr

Highest degree: PhD, Department of psychology, the University of Korea

Position title: Professor, Department of Psychology, Chungnam National University

Areas of interest: Brain Science, Neuroscience, Electro Physiology 